\title{
L’impossible colonisation du Japon pendant son « siècle chrétien »
}

\author{
Nathalie Kouamé
}

Avertissement: La transcription des termes japonais est faite selon le système Hepburn. Conformément à la tradition du Japon, le patronyme des personnes japonaises citées apparaît avant leur nom personnel.

\begin{abstract}
« C'est alors que la clique des chrétiens est venue au Japon. Ils ne se sont pas contentés d'envoyer leurs bateaux de commerce et d'introduire leurs marchandises, ils ont aussi voulu répandre leur doctrine hérétique, troubler la religion orthodoxe, et de cette façon renverser le gouvernement de notre pays et se l'approprier. C'est là le signe avant-coureur de grands malheurs. Il faut donc interdire [la présence de ces gens, leur doctrine et leurs activités subversives]. Le Japon est le pays des dieux et le pays des bouddhas : on y révère les dieux, on y vénère les bouddhas ; on s'y consacre à la voie de l'humanité et du sens moral, on y étudie la loi du bien et du mal. Ceux qui enfreignent [ces principes] sont punis, en fonction de la gravité de leur forfait, selon les cinq châtiments: tatouage, amputation du nez, amputation des jambes, castration-confinement, peine capitale. $\gg$

Ishin Sûden, Bateren tsuihô no bun [De l'expulsion des missionnaires chrétiens], $23^{\mathrm{e}}$ jour du $12^{\mathrm{e}}$ mois de la $18^{\mathrm{e}}$ année de l'ère Keichô ( $1^{\text {er }}$ février 1614$)^{1}$.
\end{abstract}

« N'exploite pas le monde qui veut. » Par cette formule passablement provocante, Fernand Braudel exprimait l'idée que la révolution industrielle anglaise avait certes été rendue possible par «l'exploitation impérialiste du monde », mais qu'elle procédait tout autant des structures socio-économiques internes de l'Angleterre' ${ }^{2}$. Avec cette acception, l'expression s'applique donc à bien d'autres cas historiques d'essor économique et de domination à l'échelle mondiale, par exemple celui de la dilatation des monarchies ibériques au $\mathrm{XVI}^{\mathrm{e}}$ siècle, où là aussi c'est au sein des structures politiques, économiques, sociales et culturelles des deux métropoles espagnole et portugaise qu'il faut chercher une partie des facteurs qui en ont favorisé, puis contrarié l'expansion coloniale planétaire.

N'est pas exploité qui n'y consent. Mise à l'envers, l'aphorisme de Braudel gagne encore en valeur expressive et heuristique, en permettant cette fois-ci de considérer les faits du point de vue des parties du monde qui sont la cible de convoitises extérieures ; elle signifie que les sociétés peuvent présenter à un moment donné de leur histoire des structures qui facilitent, voire invitent leur prise de contrôle par l'extérieur, tandis qu'à d'autres périodes elles sont à même de présenter, ponctuellement, une configuration qui rend difficile, voire impossible, toute ingérence.

\footnotetext{
${ }^{1}$ Ebisawa A., H. CIESLIK, DoI T., ÔTSUKA M. (éd.), Kirishitan sho, haiya sho [Textes chrétiens et textes antichrétiens du Japon], Tôkyô, Iwanami shoten, 1970, p. 420.

${ }^{2}$ Fernand BRAUDEL, La dynamique du capitalisme, Paris, Flammarion, 1988, p. 114.
} 
L'histoire du Japon illustre à merveille notre nouvelle maxime. En effet, ce pays sut à deux reprises rendre vaines les postures impérialistes des nations occidentales : une première fois aux XVI ${ }^{\mathrm{e}}$ et XVII ${ }^{\mathrm{e}}$ siècles, lorsque les Portugais (1542 ou 1543), les Espagnols (1584), puis les Hollandais et les Britanniques (1600) vinrent se livrer dans l'archipel à des activités religieuses et/ou économiques; une seconde fois au XIX ${ }^{\mathrm{e}}$ siècle, quand les Américains contraignirent les shôgun à ouvrir les ports nippons (1853-1854), point de départ d'une intense «modernisation »-occidentalisation organisée à partir de 1868 par un «gouvernement éclairé » (Meiji).

Nous évoquerons ici la première de ces rencontres entre Japon et Occident. Elle se distingue de la seconde sur au moins trois points. D'une part, elle fut tout à fait spontanée : c'est semble-t-il à la suite du naufrage d'une poignée de Portugais sur l'île de Tanegashima, à l'extrême sud du Japon, que s'instaurèrent des liens réguliers entre les Japonais et les Européens, ces derniers étant pourtant présents en Asie orientale depuis les années 1510. D'autre part, du fait du système du patronat et du dynamisme de la jeune Société de Jésus, les faits de religion ont occupé dans ces tout premiers rapports une place centrale ; Charles R. Boxer a justement qualifier la période de «siècle chrétien » $(1549-1650)^{3}$. Le « fruit » acquis par les missionnaires semble de fait avoir été important : l'évêque du Japon Luis de Cerqueira (1552-1614) avançait en 1602 le chiffre de trois cent mille convertis pour une population japonaise de dix à douze millions d'habitants, ce qui représente un taux d'évangélisation supérieur aux deux cent mille fidèles chrétiens acquis un demi-siècle plus tard dans un empire chinois au moins quatre fois plus peuplé que le Japon ${ }^{4}$. En plus de ces estimations plus ou moins fiables, on doit bien entendu tenir compte de tous les effets non quantifiables du passage des missionnaires dans l'archipel : introduction de conceptions religieuses inédites, essor de nouvelles formes ou thématiques artistiques, apport de techniques inconnues, changements dans les perceptions de la réalité de l'échiquier politique mondial, etc.

Troisième et dernière caractéristique de ces primes relations nippo-occidentales, elles furent alors incontestablement à l'avantage du Japon : à l'aube des Temps modernes, ce sont les Japonais qui ont fixé les règles du jeu, non les Occidentaux. Ce n'est pourtant pas faute, de la part de ces derniers, de comportements coloniaux. En effet, les missionnaires du Japon ont pu représenter de la façon la plus officielle les intérêts des États européens aux tendances alors expansionnistes ${ }^{5}$. Les jésuites en particulier, qui ont pendant longtemps gardé le monopole de la mission japonaise, ont montré dans l'archipel le même désir de « conquête spirituelle » que celui qu'ils manifestaient ailleurs ; ils y ont également établi un comptoir, à Nagasaki, y ont participé à des trafics d'armes voire d'esclaves japonais, et sont intervenus dans les conflits politiques locaux. Il reste que rien, dans la configuration du Japon de l'époque, n'aurait permis l'existence d'un binôme évangélisation-colonisation. Ou plus exactement : ce binôme, qui avait été imposé aux Amériques et aux Philippines, prit au pays du Soleil levant un tour tout à fait inattendu, puisque les Japonais finirent par suivre euxmêmes la Voie des conquistadors.

\footnotetext{
${ }^{3}$ Charles R. Boxer, The Christian Century in Japan, 1549-1650, Berkeley, University of California Press, 1974

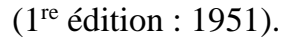

${ }^{4}$ Ce chiffre est avancé par Joseph Dehergne dans l'entrée « Jésuites en Chine » du Dictionnaire de la civilisation chinoise, Paris, Encyclopædia Universalis et Albin Michel, 1998, p. 369.

${ }^{5}$ On sait par exemple que François Xavier était tout autant au service de Jean III (1502-1557), roi de Portugal, qu'à celui d'Ignace de Loyola (1491-1556) et du pape Paul III (1468-1549). Après lui, le jésuite Alexandre Valignano (1539-1606) se présenta un temps au Japon avec le titre d'ambassadeur du vice-roi des Indes de Goa (1591). Autre exemple des liens étroits entre missionnaires du Japon et pouvoirs politiques d'Europe, plusieurs Franciscains ont engagé des pourparlers avec des dirigeants japonais au nom des autorités espagnoles des Philippines : Blazquez Pedro Bautista (1546-1597) avec le général Toyotomi Hideyoshi (1537-1598) en 1593, Jerónimo de Jesús de Castro (†1601) en 1601 avec le futur shôgun Tokugawa Ieyasu (1542-1616).
} 
Mais examinons les faits qui expliquent que le Japon ait gardé une indépendance absolue à l'heure de la première mondialisation.

\section{Première époque : une entente relative (1549-1579)}

On pourrait distinguer trois périodes successives dans l'histoire des relations entre les missionnaires chrétiens, les pouvoirs locaux du Japon et les pouvoirs coloniaux européens de l'Asie orientale.

La première époque s'ouvre en 1549 par l'arrivée de François Xavier à Kagoshima et s'achève en 1579 par les premiers pourparlers autour de la cession en faveur des jésuites du port de Nagasaki. Au cours de ces trois décennies la convergence des intérêts respectifs des parties créa entre elles un climat d'entente relative qui autorisa l'arrivée continue de missionnaires européens et l'accroissement du nombre de chrétiens nippons.

De fait, pendant cette première période, c'est une soixantaine de Jésuites qui séjournèrent un temps, ou bien s'installèrent définitivement au Japon. Portugais pour une bonne part, surtout dans les premières années, ces missionnaires s'activèrent principalement dans la grande île méridionale de Kyûshû, où se trouvaient la plupart des ports du commerce international (Hakata, Funai, Hirado...), et secondairement dans la région de Kyôto, qui était à la fois le centre géographique et le cœur symbolique du pays, puisque c'est là que se trouvaient la cour de l'empereur et celle du shôgun. Dans ces deux foyers du prosélytisme, le christianisme toucha plusieurs classes et couches sociales : de grands samurai et leurs vassaux, mais aussi leurs paysans, puisque les conversions des grands entrainaient presque automatiquement celles de leurs sujets civils ou militaires. Se convertirent également des citadins et quelques membres de la cour impériale ${ }^{6}$.

La naissance de la mission japonaise fut contemporaine des premières relations commerciales entre Européens et Japonais. En effet, François Xavier, qui pour sa part conçut le projet de fonder un comptoir dans la ville alors prospère de Sakai (non loin de Kyôto), ne fit que suivre les pas des marchands portugais arrivés au Japon quelques années avant lui ${ }^{7}$ et retenus dans ce pays par les attraits économiques qu'il présentait. De fait, à une époque où la mine d'argent du Potosí, toute juste découverte en 1545, était encore loin d'être exploitée à son maximum, le Japon offrait lui aussi bien des promesses en matière de métaux précieux : quand les Européens arrivèrent dans l'archipel, on venait tout juste d'y mettre en œuvre de nouvelles techniques d'affinage du métal argent (1533) et de découvrir de nouveaux et riches gisements, en particulier celui d'Iwami, dans le sud-ouest de l'île principale de Honshû. François Xavier nous apprend du reste que les Espagnols qui rôdaient alors dans les parages appellaient les îles du Japon Platarias, du mot plata qui signifie « argent ». Cette appellation était alors largement justifiée par le fait que l'archipel était déjà devenu l'un des premiers

\footnotetext{
${ }^{6}$ Pour se faire une idée générale de l'histoire du premier christianisme au Japon, on consultera en anglais l'ouvrage déjà cité de Charles R. Boxer et en japonais le « Grand dictionnaire de l'histoire du christianisme au Japon » (Nihon kirisutokyô rekishi daijiten) des Éditions Kyôbunkan, 1988, ainsi que l'ouvrage dirigé par Hubert CIESLIK et ÔTA Yoshiko, Kirishitan [Les premiers chrétiens du Japon], Tôkyô, Tôkyôdô shuppan, 2001 (1 ${ }^{\text {re }}$ édition : 1999). En français, voir Minako DEBERGH, «Premiers jalons de l'évangélisation de l'Inde, du Japon et de la Chine », J.-M. MAYeur, C. PIETRI, A. VAuChez et M. Venard (dir.), Histoire du christianisme (8). Le temps des confessions (1530-1620), Paris, Desclée, 1992, p. 787-853; Pierre DUNOYER, Histoire du catholicisme au Japon, 1543-1945, Paris, Les Éditions du Cerf, 2011 ; N. KouAME (dir.), « La première évangélisation du Japon (XVI ${ }^{\mathrm{e}}-\mathrm{XVII}{ }^{\mathrm{e}}$ siècles). Perspectives japonaises », Histoire \& Missions chrétiennes (Karthala), $\mathrm{n}^{\circ} 11$, septembre 2009, p. 5-163 ; N. KoUAME, Le christianisme à l'épreuve du Japon médiéval, ou les vicissitudes de la première mondialisation, Paris, Karthala, 2014.

${ }^{7}$ En se fondant sur deux sources historiques, l'une rédigée en portugais, le Traité des découvertes du monde d'Antonio Galvao (1563), l'autre écrite en sino-japonais, Teppô-ki (1606), « Histoire de l'introduction au Japon des armes à feu », les spécialistes admettent l'idée que l'arrivée des Portugais au Japon fut d'abord fortuite, puisque due à un naufrage. Cependant la date de cette arrivée, 1542 ou 1543, fait encore l'objet de discussions.
} 
producteurs et exportateurs mondiaux de ce métal : dès les années 1540 il fournissait à la Chine une partie de l'argent dont elle avait besoin pour son système économique et fiscal ${ }^{8}$.

N'était-ce pas là, a priori, une bonne raison de convoiter un pays qui, par ailleurs, pouvait paraître bien vulnérable ? A première vue, le Japon était en effet minuscule et peu peuplé en comparaison des mondes indien et chinois. Mieux encore, le pays était plongé depuis plus d'un demi-siècle dans une guerre civile où le pouvoir appartenait à ceux qui le prenaient et le conservaient par la force des armes. Enfin, les jésuites commençaient à pénétrer au Japon, lentement mais sûrement, dans l'espoir de sauver les âmes, au nom du Christ, des rois et des papes d'Europe. Mais non, décidément : en ce milieu de $\mathrm{XVI}^{\mathrm{e}}$ siècle, tout concourait à rendre le territoire nippon inexpugnable.

Primo, le Japon ne fut pas colonisé pour cette raison extrêmement simple - mais cruciale qu'il n'y eut au départ aucun projet de conquête de l'archipel, un projet à la Cortés ou la Pizarro par exemple. C'est que, dans les Indes orientales, du moins pendant les deux premiers tiers du $\mathrm{XVI}^{\mathrm{e}}$ siècle $^{9}$, les Portugais ne visèrent ni l'acquisition de vastes territoires ni le peuplement systématique des zones conquises. Quant aux Espagnols qui occupèrent les Philippines à partir des années 1560, rien ne laisse penser qu'ils ont eu des visées précises sur le Japon ${ }^{10}$. Cela dit, au début du XVII ${ }^{\mathrm{e}}$ siècle, l'argument d'une menace européenne a bel et bien été employé par les potentats nippons pour justifier l'expulsion du pays des «barbares du sud » (jap. nanban) ainsi que la proscription de leur religion ; c'est ce qu'indique par exemple le texte que nous avons cité en exergue et qui fut rédigé en 1614 par l'une des éminences grises du premier shôgun Tokugawa pour justifier l'expulsion définitive du Japon des missionnaires chrétiens.

Secundo, il faut bien admettre que l'attitude des Japonais à l'égard des étrangers aurait rendu difficile toute intrusion de ces derniers. Sur ce point, il faut relire attentivement François Xavier qui, tout en louant la curiosité intellectuelle et l'aptitude à la « raison » de la population japonaise, brosse aussi le portrait d'un peuple ayant la plus haute opinion de luimême. C'est peut-être à cet orgueil qu'il fait allusion lorsqu'il avoue dans une lettre adressée à Ignace de Loyola que : «Jamais je ne pourrai écrire tout ce que je dois aux gens du Japon car c'est à leur contact que Dieu notre Seigneur m'a donné la connaissance approfondie de mes dispositions infinies au mal ${ }^{11}$. »Il est certain qu'à cette époque les Japonais avaient déjà une idée très nette de leur unité et originalité culturelles. L'existence de ce sentiment qu'on peut qualifier de «national » se manifestait alors notamment par la volonté des grands

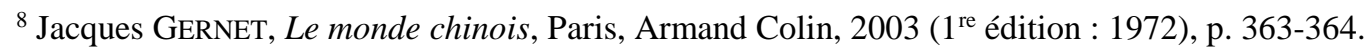

${ }^{9}$ Sur les «nouvelles orientations » des entreprises lusitaniennes dans l'Asie des années 1570-1610, voir Sanjay Subrahmanyam, L'empire portugais d'Asie, 1500-1700. Histoire politique et économique, Paris, Maisonneuve \& Larose, 1999, p. 141-184.

${ }^{10}$ De nos jours, subsiste un cliché historiographique selon lequel, à l'occasion du naufrage du navire espagnol San Felipe dans la province de Tosa à la fin d'octobre 1596, les autorités japonaises auraient été informées par l'équipage des visées impérialistes de l'Espagne de Philippe II et du rôle joué ordinairement par les missionnaires dans la préparation des opérations des conquistadors; leurs propos auraient déterminé ces autorités à exécuter vingt-six chrétiens à Nagasaki en février 1597 ; parmi ces victimes se trouvait l'ambassadeur de Manille, le franciscain Pedro Bautista (1546-1597). En vérité cependant, le lien de cause à effet entre, d'une part, les interrogatoires de l'équipage lors de l'enquête et, d'autre part, l'exécution publique dans la cité chrétienne n'est pas clair, et dans tous les cas on peut croire que les autorités du Japon n'avaient pas attendu le naufrage du San Felipe pour subodorer des dispositions impérialistes chez les Espagnols qui occupaient les Philippines depuis les années 1560. Ajoutons qu'un seul des membres de l'équipage du navire naufragé est à compter parmi les victimes de février 1597. Pour plus de détails sur cette affaire et sa signification réelle, cf. N. KOUAME, «Une "drôle de répression". Pour une nouvelle interprétation des mesures antichrétiennes du général Toyotomi Hideyoshi (1582-1598) », A. BROTONS, Y. BRUnETON, N. KoUAME (dir.), Etat, religion et répression en Asie. Chine, Corée, Japon, Vietnam. XIII - XXI ${ }^{e}$ s., Paris, Karthala, 2011, p. 170-176.

${ }^{11}$ Lettre du 29 janvier 1552, François XAVIER, Correspondance 1535-1552, Lettres et document. Traduction intégrale, présentation, notes et index de Hugues Didier, Paris, Desclée de Brouwer Bellarmin, 2005 ( $1^{\text {re }}$ édition : 1997), p. 382.
} 
samurai (daimyô) de laisser en place les institutions impériale et shôgunale qui entretenaient la fiction d'un État japonais immuable commandé par un grand prêtre - celui que notre époque contemporaine appelle «l'empereur du Japon»- vu comme le descendant de la déesse shintô Amaterasu Ômikami. En vérité toutefois, cela faisait des siècles que les empereurs en titre ne gouvernaient plus ; quant aux shôgun, leurs délégués, ils étaient depuis longtemps à la merci d'une destitution ou d'un assassinat orchestrés par l'un ou l'autre de ces daimyô.

Troisième facteur assurant l'étanchéité de l'archipel, les données sociales de l'époque ont largement joué pour empêcher que les marchands et les missionnaires européens, au demeurant fort peu nombreux ${ }^{12}$, puissent représenter un réel danger. Dans ce Japon-là, en effet, toute la population était armée : les paysans, les citadins, les hommes de religion étaient en armes, tout comme bien entendu ceux que l'on appelait les samurai, soit les guerriers qui continuaient à dominer la société, qu'ils oeuvrent pour leur compte, ou qu'ils se retrouvent associés aux puissantes ligues paysannes ou religieuses du moment. Il n'est donc guère étonnant que cette nation diversement armée de sabres, d'arcs et de lances, voire d'outils agricoles, et pour l'heure tout entière engagée dans des conflits militaires, ait montré le plus grand intérêt pour les armes à feu des Portugais.

Il existe plusieurs thèses relatives à l'introduction au Japon des mousquets (esp. espingarda, jap. teppô) : les spécialistes ne sont d'accord ni sur la date de l'arrivée de ces armes, ni sur le profil de ceux qui les ont importées (marchands portugais ou pirates chinois), ni sur les circuits de leur diffusion dans le pays ${ }^{13}$. Mais une chose reste absolument certaine : sitôt ces armes arrivées au Japon, au plus tard en $1543^{14}$, elles ont été fabriquées sur place, dans plusieurs centres de production (Tanegashima, Bônotsu, Hirado, Sakai...). Et, bien entendu, on s'est tout autant empressé d'en apprendre l'usage et d'en équiper les troupes : les «bandes de Negoro » (jap. Negoro-shû), qui représentaient le bras armé du puissant temple Negoroji au centre de l'archipel, ainsi que les soldats d'Oda Nobunaga (1534-1582) qui ont participé à la grande bataille de Nagashino (1575) ont laissé leur nom dans l'histoire du Japon pour la modernité de leur équipement en mousquets - dont l'efficace a peut-être pourtant longtemps consisté à provoquer stupeur et tremblement dans les rangs ennemis. Dans ces conditions, il était difficile pour les Européens d'impressionner les Japonais, qui finiront du reste à la fin $\mathrm{du} \mathrm{XVI}^{\mathrm{e}}$ siècle par vendre leurs compétences militaires hors de leurs frontières, dans les diverses parties de l'Asie orientale.

Dernier facteur qui rendait improbable toute velléité de colonisation du Japon, les diverses entités politiques qui coexistaient dans le contexte de guerre civile de l'époque présentaient une réelle cohérence interne, qu'on aurait pu difficilement fragiliser car les seigneurs de la guerre reproduirent à l'échelle de leur royaume les solides modèles politiques autrefois mis en place par l'État impérial-shôgunal. Concrètement, ils développèrent l'économie de leur domaine, créèrent des villes, édictèrent des lois, eurent une politique culturelle, etc. Les

\footnotetext{
${ }^{12}$ On comptait moins de cent cinquante missionnaires lorsque fut promulgué l'édit d'expulsion de 1614.

${ }^{13}$ Sur ces questions, voir l'un des récents catalogues d'exposition du Musée national d'histoire et d'ethnologie de la ville de Sakura, Rekishi no naka no tenpô denrai. Tanegashima kara Boshin sensô made [L'introduction au Japon des armes à feu, Perspectives historiques. De Tanegashima à la guerre de Boshin], 2006.

${ }^{14}$ Cette date de 1543 est donnée par le Teppô-ki [Histoire de l'introduction au Japon des armes à feu] (1606), le premier texte rédigé par un Japonais à propos de l'arrivée des Européens au Japon. L'auteur, un moine de confession zen nommé Bunshi Genshô (1555-1620), y expose une version des faits selon laquelle les premiers Portugais à avoir foulé le sol du Japon arrivèrent dans l'île de Tanegashima en 1543 et firent connaître à cette occasion leurs armes à feu, qui furent aussitôt testées, achetées, étudiées et reproduites à Tanegashima, puis de là diffusées dans tout le pays. Il semblerait que ce récit écrit plus d'un demi-siècle après les faits ait surtout cherché à donner au clan et à l'île éponyme de Tanegashima une stature nationale de «berceau (japonais) des armes à feu » (jap. Teppô no waga Tanegashima ni kenyo nari). Cf. SHIMIZU Hirokazu, Shokuhô seiken to kirishitan. Nichiô kôshô no kigen to tenkai [Oda Nobunaga et Toyotomi Hideyoshi face aux premiers chrétiens du Japon. Origines et développement des relations nippo-européennes], Tôkyô, Iwata shoin, 2001, p. 29-88.
} 
territoires soumis aux ligues paysannes ou religieuses montraient une cohésion comparable. Mais surtout, vu le jeu permanent d'alliances (et de retournements d'alliance) qui existaient entre ces divers pouvoirs, il est permis de penser qu'à la moindre alerte nationale menaçant leurs intérêts particuliers ils auraient été capables d'engager un front commun. Ou du moins d'en avoir l'idée.

Si les marchands et les missionnaires européens ne se sont donc pas imposés au Japon à leur arrivée, il faut tout de même constater qu'ils ont réussi à s'y installer assez durablement.

Dans ce processus, les jésuites ont joué d'emblée un double rôle : d'une part ils ont servi d'intermédiaires entre les Japonais et les marchands portugais qui faisaient des affaires ici et là dans le sud de l'archipel; d'autre part, ils se sont très vite transformés en entrepreneurs oeuvrant pour leur propre compte. Dès 1556 , les membres de la Société de Jésus utilisèrent la fortune de l'un de leur coreligionnaire pour investir dans le commerce de la soie grège chinoise, le produit-phare des importations japonaises jusqu'au milieu du XVII ${ }^{\mathrm{e}}$ siècle.

Pendant cette première époque, il est arrivé plusieurs fois que les «soldats du Christ» entrent en conflit avec les potentats locaux, que ceux-là soient des laïcs ou des ecclésiastiques. Ces heurts se sont parfois produits pour des raisons idéologiques, lorsque par exemple des moines bouddhistes considéraient le message chrétien comme incompatible avec la voie du bouddha. Ce cas de figure est illustré par l'une des premières disputations nippo-européennes, celle qui opposa à l'automne 1551 dans la province de Bungo François Xavier au moine «Fucarandono»: les deux clercs entreprirent alors pendant plusieurs jours d'affilée un dialogue de sourd qui ne se termina de manière pacifique que parce que le « roi de Bungo », qui assistait aux débats avec ses vassaux, avait constamment veillé à protéger le Navarrais qui représentait tout autant la chrétienté que la petite communauté de marchands portugais s'activant depuis peu dans les parages ${ }^{15}$. Une dizaine d'années plus tard, en 1561 , l' « incident de Miyanomae » illustrait encore plus clairement la manière dont les questions économiques et les enjeux religieux déterminaient ensemble le ton des relations entre samurai et missionnaires. En effet, après l'assassinat cette année-là de quatorze marins portugais par des Japonais à l'occasion d'un différend concernant la vente d'étoffes de soie, le seigneur de la localité Matsura Takanobu (1529-1599) paya fort cher le retour des navires de commerce étrangers dans son port de Hirado: non seulement il dut présenter des excuses aux pères jésuites, mais en outre il lui fallut autoriser la reconstruction de l'église qu'il avait détruite deux ans auparavant en réaction aux actes iconoclastes des chrétiens à l'encontre d'images et de soutras bouddhiques; dès 1565 , les rapports se tendirent à nouveau entre Takanobu et les Portugais, et le christianisme redevint officiellement interdit dans le domaine des Matsura ${ }^{16}$.

En dehors de la possibilité d'intégrer les circuits commerciaux internationaux, les activités des missionnaires étrangers au Japon pouvaient représenter bien d'autres avantages, ou désavantages, aux yeux des pouvoirs locaux : en 1580, le soutien logistique en balles, poudre, vivres et argent apporté par le jésuite Alexandre Valignano (1539-1606) à Arima Harunobu (1561 ?-1612) permit à ce dernier, fraîchement baptisé, de protéger son territoire des visées expansionnistes de son rival Ryûzôji Takanobu (1529-1584) et de la rébellion de certains de

\footnotetext{
15 Bien que contesté par l'historiographie contemporaine, le témoignage de Fernão Mendes Pinto sur cette disputation de 1551 est assez vraisemblable. Cf. Fernão Mendes PINTO, Pérégrination. Récit de voyage traduit du portugais et présenté par Robert Viale, Paris, La Différence, 2002 (1 ${ }^{\text {re }}$ édition : 1991), p. 848-875.

${ }^{16}$ Cf. Seno S., Shinkawa T., SAEKi K., Gonoi T., KomiYa K. (dir.), Nagasaki ken no rekishi [Histoire du département de Nagasaki], Tôkyô, Yamakawa shuppansha, 1998, p. 126-128.
} 
ses vassaux ; en contrepartie, sur la demande des jésuites, Harunobu détruisit la totalité des temples bouddhiques et sanctuaires shintô de son domaine ${ }^{17}$.

De façon générale, les occupations temporelles des missionnaires chrétiens expliquent donc largement l'intérêt que les pouvoirs nippons ont montré à leur égard. Très rapidement, une sorte de relation contractuelle semble même s'être instaurée entre certains pères étrangers et certains daimyô, notamment dans l'île méridionale de Kyûshû : aux jésuites d'attirer dans les ports les navires portugais, aux seigneurs locaux de laisser les missionnaires mener leurs activités religieuses. C'est dans ce cadre-là que l'on vit certains guerriers d'importance accepter d'être baptisés. La première conversion d'un potentat date de 1563 ; c'était un samurai important de l'île de Kyûshû, qui s'appelait Ômura Sumitada (1533-1587) ; son entrée dans la religion catholique fut suivie de peu par l'ouverture dans son domaine du tout nouveau (mais éphémère) port de commerce international de Yokose.ura. La ville portuaire de Nagasaki fut créée dans les mêmes conditions en 1570, dans une autre partie du domaine dudit Ômura. Cela dit, les conversions de grands samurai n'ont pas toutes été obtenues de cette manière: des guerriers comme Takayama Ukon (1552-1615) ou Konishi Yukinaga $(† 1600)$ ne peuvent être soupçonnés d'opportunisme ; les deux moururent chrétiens, l'un en exil, l'autre sur l'échafaud.

$\mathrm{Au}$ cours de cette première époque, l'éclatement du paysage politique du Japon fut à l'avantage des marchands et des prêtres étrangers. De fait, lorsque ces derniers entraient en conflit avec un seigneur nippon et se trouvaient expulsés de la zone qu'ils avaient investie, il leur était toujours possible de reprendre leurs activités dans une autre région du Japon. En revanche, dans la période qui suivit, la réunification politique du pays restreignit considérablement leur marge de manœuvre.

\section{Deuxième époque : tensions (années 1580-1614)}

Dès les années 1580, de vives tensions apparurent entre les missionnaires, les pouvoirs locaux et les pouvoirs coloniaux européens, sans toutefois que l'existence de la mission japonaise ou la sécurité même des étrangers au Japon s'en trouvassent remises en cause.

Ce tournant correspond à l'apparition dans l'archipel d'un pouvoir politique fort, en l'occurrence celui du général Toyotomi Hideyoshi (1537-1598), qui fut le premier samurai à prendre le contrôle de l'ensemble du pays après plus d'un siècle de guerres civiles ${ }^{18}$. Au cours d'une fulgurante conquête du Japon (1582-1590), Hideyoshi récupéra l'œuvre politique léguée par son seigneur Oda Nobunaga (1534-1582) tout en multipliant les grandes campagnes militaires qui lui assurèrent successivement la possession du Centre, du Sud-Ouest (île de Shikoku : 1585, île de Kyûshû : 1587), et enfin du Nord-Est (1590) du pays.

D'emblée, Hideyoshi montra toutes les qualités d'un grand homme d'État ${ }^{19}$. Ainsi, pour entériner les nouveaux rapports féodaux nés de la guerre civile, il obligea les seigneurs soumis à cadastrer leurs terres, redéfinissant à cette occasion les principes agraires et fiscaux qui commandaient leurs rapports avec les paysans; il a battu monnaie ; il a freiné la mobilité sociale de l'époque, celle-là même qui l'avait conduite au sommet du pouvoir, en cherchant à

\footnotetext{
${ }^{17}$ Ibid., p. 133, et Nihon kirisutokyô rekishi daijiten [Grand dictionnaire de l'histoire du christianisme au Japon], op. cit., p. 68. Sur les relations entre jésuites, samurai et bonzes à l'aube de la mission japonaise, on trouvera quantité d'exemples dans le bel ouvrage de Léon BouRdon, La Compagnie de Jésus et le Japon, 1547-1570, Lisbonne-Paris, Centre culturel portugais de la Fondation Calouste Gulbenkian, 1993.

${ }^{18}$ En anglais l'ouvrage de référence sur ce personnage historique de toute première importance reste celui de Mary Elisabeth BERRY, Hideyoshi, Cambridge Mass.-London, Harvard University Press, 1982.

19 Rappelons une fois pour toutes que, bien qu'il fût gratifié par la cour impériale du Japon de titres et de fonctions très élevés, Hideyoshi ne fut jamais nommé shôgun. La charge resta d'ailleurs vacante de 1573 (date à laquelle le dernier shôgun Ashikaga fut chassé du pouvoir par Oda Nobunaga) à 1603 (lorsque Tokugawa Ieyasu la reçut de l'empereur Goyôzei).
} 
désarmer les paysans et les religieux. Mais, du point de vue qui nous préoccupe, il fit encore beaucoup mieux : Hideyoshi a aussi très tôt conçu des projets impérialistes à l'égard des pays de l'Asie orientale.

Dans cette politique radicalement nouvelle (les dernières interventions militaires du Japon sur le continent remontaient au $\mathrm{VII}^{\mathrm{e}}$ siècle $^{20}$ ), notre homme se montra pour le moins ambitieux : dès 1585, il exprimait le désir d'envahir la Chine! Ruse d'un vieux guerrier soucieux de détourner les énergies de samurai trop fraîchement soumis et d'une masse de rônin ${ }^{21}$ fonctionnant en électrons libres, ou délire mégalomaniaque d'un homme qui ne sentait plus sa force ? Le projet de Hideyoshi fut concrétisé en $1592^{22}$. La Corée devant offrir un couloir d'accès au continent, les armées japonaises s'attaquèrent d'abord à la péninsule. Les sources japonaises parlent d'un corps expéditionnaire s'élevant à 160000 soldats. Quelques jours après sa première grande victoire éclair à Séoul, due tout autant au nombre considérable d'hommes déployés qu'à l'usage des mousquets et à l'impréparation des Coréens, Hideyoshi annonçait dans une « lettre au sceau rouge » adressée à son neveu et héritier officiel, le Grand Chancelier Hidetsugu (1568-1595), les dispositions qu'il faudrait prendre une fois le continent conquis :

«Sa Majesté 1'Empereur [du Japon, soit Goyôzei, r. 1586-1611] sera transférée dans la capitale chinoise [Pékin]. Dès à présent, on doit s'atteler aux préparatifs de ce transfert qui aura lieu dans deux ans. On lui attribuera dix provinces ${ }^{23}$ autour de la capitale chinoise. Au sein de ces dix provinces, chacun des nobles de la cour se verra accorder un fief. A la cour, les personnes de basse condition sont dix fois plus nombreuses; on accordera des grâces, selon leurs qualités personnelles, à celles d'entre elles qui sont les mieux placées hiérarchiquement.

En ce qui concerne le titre de Grand Chancelier de Chine, Sa Seigneurie [soit Hideyoshi] l'accorde à Hidetsugu, comme mentionné ci-dessus. On vous attribuera donc cent provinces autour de la capitale chinoise. Pour ce qui est de la personne qui doit occuper la fonction de Grand Chancelier du Japon, Sa Seigneurie choisira selon son inclination entre le Conseiller Secondaire de Yamato [Hashiba Hideyasu, autre neveu de Hideyoshi, frère de Hidetsugu] et l'Auditeur de Bizen [Ukita Hide.ie, vassal de Hideyoshi].

Celui qui dans l'archipel aura le rang d'Empereur du Japon sera soit le Jeune Prince [Yoshihito, fils de l'empereur Goyôzei] soit le Seigneur de la Huitième Avenue [Toshihito, frère cadet de Goyôzei] ${ }^{24}$. »

Le fait d'imaginer ainsi que le Japon pourrait s'emparer d'un empire peuplé de quatrevingts ou cent millions d'âmes, tout comme le rêve de dédoubler systématiquement les fonctions suprêmes d'une future gouvernance sino-japonaise et le désir de concurrencer

\footnotetext{
${ }^{20} \mathrm{Ce}$ fut la bataille navale qui se déroula en 663 au sud-ouest de la péninsule coréenne, près de Paekkang, et qui opposa la flotte nippo-coréenne des cours du Yamato (Japon) et de Paekche, aux forces sino-coréennes des Tang et du royaume de Silla. Il va de soi que les deux expéditions mongoles qui ont ébranlé le Japon en 1274, puis 1281 ne peuvent être rangées dans la catégorie des interventions extérieures de ce pays. De même, étant par définition illégale, l'activité de ceux que l'on appelait en Extrême-Orient depuis le XIV siècle les "pirates japonais », ne relève pas des relations d'Etat à Etat, même si les raids opérés sur les côtes et dans l'arrière-pays du continent ont souvent déterminé la teneur des relations officielles entre Japon, Chine et Corée.

${ }^{21}$ Littéralement «homme flottant », c'est-à-dire sans maître et sans emploi. Les conflits militaires de l'époque des guerres civiles et de la réunification politique du Japon accrurent considérablement le nombre de ces samurai.

${ }^{22}$ Deux ouvrages récents font le point sur le déroulement de cet épisode historique : KITAJIMA Manji, Hideyoshi no Chôsen shinryaku [L'agression de la Corée par Hideyoshi], Tôkyô, Yamakawa shuppansha, 2002, et Stephen TuRnBull, Samurai Invasion. Japan's Korean War, 1592-1598, Londres, Cassell, 2002.

${ }^{23}$ Est-il question ici des sous-préfectures (en chinois : xian) du système administratif chinois ?

${ }^{24}$ Lettre du $18^{\mathrm{e}}$ jour du $5^{\mathrm{e}}$ mois de la $20^{\mathrm{e}}$ année de l'ère Tenshô (1592), éditée dans KoDAMA Kôta, SASAKI Junnosuke (dir.), Shiryô ni yoru Nihon no ayumi, kinsei hen [L'évolution historique du Japon vue à travers ses sources, volume d'histoire moderne], Tôkyô, Yoshikawa kôbunkan, 2001 (1 ${ }^{\text {re }}$ édition : 1996), p. 18.
} 
l'autorité sacrée de l'empereur de Chine par celle de son homologue japonais étaient proprement extravagants à une époque où la dynastie des Ming, alors représentée par Wanli (1563-1620), restait la première puissante de l'Extrême-Orient malgré la crise démographique, financière et politique que connaissait l'empire. L'entreprise de Hideyoshi, renouvelée en 1597, se solda par un désastre, et pour les Japonais, qui durent finalement se retirer de la péninsule en 1598 (après la mort de Hideyoshi) devant la résistance sinocoréenne $^{25}$, et pour les Coréens, qui, massacrés ou déportés, endurèrent les horreurs de la guerre et frisèrent la « catastrophe $»^{26}$ démographique.

En comparaison, les autres prétentions internationales de Hideyoshi paraissent presque modérées. De fait, au début des années 1590, notre homme signifia plusieurs fois aux autorités de Manille qu'elles lui devaient soumission et tribut; il formula les mêmes exigences à l'égard de Taïwan. Par ailleurs, sa volonté d'affermir son contrôle sur le Nord-Est du Japon ainsi que la perspective d'avoir accès à la Corée et à la Chine en passant par une route du Nord le conduisirent à renforcer la stature politique du clan des Kakizaki basé à Fukuyama (extrêmité sud de l'actuelle Hokkaidô) en lui accordant en 1593 le droit de percevoir des taxes sur la navigation marchande au sein de la vaste région qui était à l'époque nommée Ezogashima (ou Ezo), et qui correspond à peu près à l'ensemble insulaire constitué aujourd'hui par Hokkaidô, Sakhaline et $\operatorname{Kouriles}^{27}$. Or le fait que ces «îles barbares » ${ }^{28}$ étaient principalement habitées par des Aïnous, un peuple qui possédait une langue et une culture propres et qui avait jusqu'alors réussi à conserver une relative indépendance vis-à-vis du Japon ${ }^{29}$, donnait une dimension clairement impérialiste aux prétentions de Hideyoshi et de ses nouveaux vassaux du Nord.

Il faut croire qu'une telle disposition d'esprit finit par sembler tout à fait naturelle au Japon : une fois Hideyoshi disparu (1598), les guerriers Tokugawa furent eux aussi tentés par une politique extérieure agressive. En 1609, ils approuvèrent l'invasion de l'archipel méridional des Ryûkyû par le clan des Shimazu de Kyûshû ; en 1630, au prétexte que Manille était devenue depuis 1614 la base de départ vers le Japon de missionnaires clandestins, ils encouragèrent Matsukura Shigemasa ( ?-1630), un seigneur dudit Kyûshû, dans son projet d'envahir la cité espagnole ; enfin, dès leur arrivée au pouvoir, ils confirmèrent les droits exclusifs sur le commerce avec Ezo du clan des Kakizaki (renommés Matsumae), et entamèrent ainsi leur lente, mais sûre domination sur un peuple aïnou qui devait par la suite se soulever plusieurs fois contre la colonisation partielle dont il fut victime, la grande révolte conduite par Shakushain en 1669 constituant l'épisode militaire le plus marquant de cette résistance, mais non le point final.

Car, bien entendu, cet expansionnisme concernant les extrêmités sud et nord de l'archipel eut ses raisons économiques, puisque les Tokugawa et leurs grands vassaux entendirent exploiter les zones soumises; le rapport de force qui s'instaura en leur faveur dans ces périphéries leur permit notamment de tirer profit de la canne à sucre des Ryûkyû ainsi que des produits de la mer et de la force de travail des Aïnous. Mais une explication de ce type suffit-

\footnotetext{
${ }^{25}$ L'intervention des armées Ming était justifiée par le fait que la Corée était un Etat vassal de l'empire chinois.

${ }^{26}$ Nous reprenons là l'expression de Francis Macouin, qui estime à quatre millions de personnes le déficit de population engendré par cette guerre un demi-siècle après les faits, cf. F. MACOUIN, La Corée du Chosŏn, 13921896, Paris, Les Belles Lettres, 2009, p. 29-31.

${ }^{27}$ Cf. Brett L. WALKer, The Conquest of Ainu Lands. Ecology and Culture in Japanese Expansion, 1590-1800, Berkeley-Los Angeles, University of California Press, 2001, p. 17-35.

${ }^{28}$ Traduction littérale d' «Ezogashima ». Toutefois, Hideyoshi et ses contemporains ignoraient la géographie exacte de cette région et s'imaginaient qu'elle était rattachée au continent eurasiatique.

${ }^{29}$ La mainmise par l'Etat impérial-shôgunal japonais sur Ezogashima fut extrêmement fragile jusqu'à la fin du $\mathrm{XVI}^{\mathrm{e}}$ siècle; à cette époque, seule la pointe méridionale de cet ensemble, autrement dit l'extrême sud de Hokkaidô, était occupée par des Japonais.
} 
elle réellement à penser le caractère tout à fait inédit de l'attitude coloniale des pouvoirs japonais au tournant des $\mathrm{XVI}^{\mathrm{e}}$ et XVII ${ }^{\mathrm{e}}$ siècle, en plein « siècle chrétien »?

Pour notre part, nous serions tentée de croire que ce premier impérialisme nippon fut en partie inspiré par la conduite des Européens en Asie orientale et dans le monde. Comme nous l'avons déjà suggéré, ce n'est pas l'histoire du Japon qui pouvait constituer un modèle en matière de politique expansionniste. Certes, le passé de la Chine impériale fournissait davantage de précédents : l'empereur Wudi de la dynastie des Han (140-87) ou l'empereur Taizong des Tang (598-649) avaient été de grands conquérants ; les premiers souverains de la dynastie des Ming (1368-1644), Hongwu et plus encore Yongle, avaient eux-mêmes bâti leur empire sur la conquête (ou la reconquête) de terres et sur la construction d'un réseau international de pays vassaux ${ }^{30}$. Sur ce dernier point, notons que réclamer tribut à Manille, comme l'avait fait Totoyomi Hideyoshi, revenait à suivre la conception chinoise des relations extérieures, selon laquelle une nation «civilisée » pouvait imposer une telle condition à des peuples «barbares », qui en contrepartie recevaient le droit de commercer avec elle. Des années 1400 aux années 1540, les Japonais s'étaient eux-mêmes pliés à cette exigence et en avaient tiré grand profit sur le plan économique. Il se peut donc que Hideyohi ait tout simplement cherché à officialiser - «à la chinoise »- les relations commerciales qui se développaient depuis quelques décennies entre les archipels nippon et philippin. Cela dit, son projet de conquête de la Chine sortait du cadre de ce schéma diplomatique qui ne présupposait pas de mainmise militaire de la nation suzeraine sur les pays tributaires. Au XVI ${ }^{\mathrm{e}}$ siècle, les Ming percevaient encore le tribut de quelques peuples sans faire montre d'aucune vélléité expansionniste; c'est qu'ils avaient depuis longtemps, en fait dès le milieu du siècle précédent, opéré un repli (relatif), symbolisé par leurs nouvelles et Grandes Murailles. En comparaison, les Européens ont dû paraître extraordinairement agressifs aux yeux de leurs contemporains asiatiques.

Pour leur part, les potentats nippons suivaient attentivement les faits et gestes de ces nouveaux venus qui n'étaient moins arrogants qu'eux-mêmes : ils les recevaient dans leurs palais pour s'entretenir avec eux, et en faisaient à l'occasion leurs conseillers personnels; ils entretenaient une correspondance avec leurs autorités de tutelle (vice-roi des Indes de Goa, gouverneur général des Philippines); ils envoyaient des ambassades dans leurs lointaines contrées, métropoles ou colonies ${ }^{31}$; et par tous ces contacts, ils avaient connaissance des faits d'armes, de piraterie, d'exploitation économique et/ou de réduction en esclavage dont ces étrangers se rendaient coupables partout où ils passaient ou s'établissaient. On ne peut s'empêcher de penser que la fréquentation de ces «barbares » a nécessairement eu des effets sur leur conception du monde et la conduite de leurs affaires. De médiocre extraction, mais parvenus au sommet du pouvoir par leur génie militaire et politique, un Toyotomi Hideyoshi ou un Tokugawa Ieyasu n'ont-ils pas été séduits par l'intrépidité, la vitalité et la violence même de ces étrangers venus de si loin pour évangéliser, commercer, conquérir, soumettre ?

\footnotetext{
${ }^{30}$ Cf. Jérôme KeRlouEgAN, « De l'expansion au recentrement : la Chine et son monde », Patrick BOUCHERON (dir.), Histoire du monde au XV siècle, Paris, Fayard, 2009, p. 619-635.

${ }^{31}$ On sait par la correspondance de François Xavier que ces échanges diplomatiques ont existé dès le début de la mission japonaise, puisque l'Apôtre des Indes note dans sa fameuse lettre datée du 29 janvier 1552 que le « duc de Bungo [Ôtomo Yoshishige] est un très grand ami des Portugais [...]. Il est informé de la grandeur du roi du Portugal. Il écrit au Roi pour s'offrir à être son serviteur et son ami : en signe d'amitié, il lui a envoyé une armure. Il a envoyé au vice-roi de l'Inde un de ses serviteurs pour lui offrir son amitié ; c'est avec moi que celuici est venu [du Japon à Cochin] », F. XAVIER, op. cit., p. 375-376. La première ambassade nippo-européenne d'envergure (1582-1590) fut une initiative d'Alexandre Valignano ; quatre jeunes gens représentant trois daimyô chrétiens de Kyûshû voyagèrent au Portugal, en Espagne, en Italie, et furent reçus par Philippe II, puis Grégoire XIII. Une seconde grande ambassade (1613-1620) fut organisée par le seigneur (non chrétien) Date Masamune (1567-1636), qui envoya un de ses samurai, Hasekura Tsunenaga (1571-1622), accompagné du franciscain Luis Sotelo (1574-1624), en Nouvelle-Espagne, à la cour de Philippe III et à Rome.
} 
En tout cas, le contenu de la lettre que Hideyoshi adressa au vice-roi de Goa en 1591-1592 (en réponse à un courrier que ce dernier lui avait adressé en 1587$)^{32}$ donne l'impression que le Japonais eut le souci de se présenter au colon portugais en des termes qui seraient compréhensibles à ce dernier : non seulement il y définissait le Japon comme le «pays des dieux » (jap. shinkoku; kami no kuni) ${ }^{33}$ et du «shintô », autrement dit comme une contrée qui, à l'instar des pays chrétiens, était dotée d'une religion et qui, pour cette raison, n'avait point besoin d'être convertie à la voie du Christ ; mais en outre il y annonçait son intention de conquérir la Chine, ce qui le plaçait virtuellement dans une position politique supérieure à celle d'un interlocuteur confiné dans un petit comptoir situé à la périphérie du flamboyant empire d'Akbar (1542-1605). Hideyoshi joignait à sa lettre quelques cadeaux : un sabre long, un sabre court, un poignard, une hallebarde, deux armures complètes... C'est que le vice-roi de Goa avait lui-même donné le ton en 1587 en offrant au Japonais, « en signe d'amitié », des armes de son pays et deux chevaux. Nul doute : au-delà de la folie ou du calcul politique, les généraux japonais qui dilatèrent le territoire nippon étaient en phase avec le monde.

Rien n'interdit de penser que leurs postures guerrières ont pu avoir un effet dissuasif sur les conquistadors issus de la chrétienté. Un demi-siècle plus tôt, dans les années 1550, François Xavier avait déjà fait savoir en Europe que : «Les Japonais sont des gens qui ont une excellente opinion d'eux-mêmes, car il leur semble que dans les armes et dans l'esprit de chevalerie il n'y en a pas d'autres qui soient comme eux ${ }^{34}$. » À l'aube du XVII siècle, peu de temps après le retrait des troupes japonaises de Corée, la Carte complète des myriades de pays dans le monde (1602) de Matteo Ricci (1552-1610) plaçait certes la Chine au centre du monde, mais elle prenait aussi acte de la nouvelle dimension impérialiste du pays du Soleil Levant en signalant pour la première fois dans l'histoire de la cartographie mondiale l'existence d'une «mer du Japon » faisant comme contrepoids à la «mer des Ming » ${ }^{35}$, et en relevant dans ses notices que «beaucoup (de Japonais) s'initient aux armes, peu aux

\footnotetext{
${ }^{32}$ Lorsque Alexandre Valignano quitta Goa en 1588 après s'être vu confier par le vice-roi Duarte de Meneses (1537-1588) la lettre que celui-ci avait rédigée à l'attention de Hideyoshi, il n'avait pas encore connaissance de la promulgation de l'édit d'expulsion des pères chrétiens par le général nippon (1587) (voir infra). Par conséquent, lorsqu'il remit cette lettre en 1591 à son destinataire, le contenu de celle-ci était ironiquement en phase avec l'actualité puisque le vice-roi y sollicitait la bienveillance du Japon à l'égard des missionnaires. L'historien Matsuda Kiichi déduit du décalage existant entre la date du brouillon sino-japonais de cette lettre $\left(7^{\mathrm{e}}\right.$ lune de la $19^{\mathrm{e}}$ année de l'ère Tenshô, 1591) et la date de sa version portugaise définitive ( $7^{\mathrm{e}}$ lune de la $20^{\mathrm{e}}$ année de l'ère Tenshô, 1592) que Hideyoshi a eu besoin de près d'un an pour rédiger sa réponse au vice-roi, les missionnaires faisant pression sur lui pour qu'il modère le ton de ses propos. Cf. MATSUDA Kiichi, Toyotomi Hideyoshi to nanbanjin [Toyotomi Hideyoshi et les barbares du sud], Tôkyô, Chôbunsha, 2001 ( $1^{\text {re }}$ édition : 1992), p. 109-114.

${ }^{33}$ Dans la terminologie shintô, le terme générique pour désigner une « divinité », ou un « dieu », est « kami »; le mot se lit shin en sino-japonais. Le «shintô » est donc la «voie » (jap. michi, dô, tô) des « dieux » (kami, shin). Si ce terme de «shintô » était déjà très ancien au Japon lorsque Hideyoshi l'utilisa dans ses édits et épîtres, l'expression de shinkoku (pays des dieux, pays divin) était beaucoup plus récente : elle était tirée d'un classique de la littérature médiévale japonaise, le Jinnô shôtô $k i$ «Histoire de la succession légitime des divins empereurs » (1339) qui commençait par cette assertion : «Le Grand Japon est le pays des dieux. » Ecrit par l'aristocrate Kitabatake Chikafusa (1293-1354), l'ouvrage était en fait un pamphlet savant qui avait pour but de soutenir l'une des deux cours impériales japonaises en guerre entre 1336 et 1392. Par ces premiers mots, Chikafusa cherchait non pas à définir le Japon comme un pays possédant une religion propre, mais comme un Etat où les empereurs régnaient du fait de la volonté des kami; dans la suite de l'ouvrage, l'auteur proposait une nouvelle interprétation de la généalogie impériale qui était censée expliquer pourquoi seul l'un des deux empereurs rivaux de son époque était légitime. Cf. KocHI Shôsuke, Chûsei no tennô kan [La conception médiévale de l'empereur], Tôkyô, Yamakawa shuppansha, 2003, p. 25-35 et p. 65-69.

${ }^{34}$ F. XAVIER, op. cit., p. 360-361.

35 Philippe Pelletier, L'Extrême-Orient. L'invention d'une histoire et d'une géographie, Paris, Gallimard, 2011, p. 326-337.
} 
lettres $^{36} \gg$. Cette mappemonde d'inspiration sino-européenne entérinait ainsi l'image que Hideyoshi avait voulu lui-même donner d'un "pays du Japon aux arcs et flèches implacables» (jap. yumiya kibishiki Nihon-koku) capable de dominer le «pays [des mandarins] à manches longues des Grands Ming » (Daimin chôshû koku $)^{37}$.

En tout cas, pour toute la seconde moitié du $\mathrm{XVI}^{\mathrm{e}}$ siècle, on ne connaît aucun projet de conquête concernant le Japon qui ressemblât de près ou de loin à celui que le jésuite espagnol Alonso Sanchez (1547-1593) trama contre la Chine et présenta au gouverneur, à l'évêque et aux notables de Manille lors de leur conseil du mois d'avril 1586. Il était question dans cette chimère de recruter des soldats japonais comme forces auxiliaires des troupes espagnoles devant partir à la conquête de la Chine. Madrid ne donna pas suite à ce projet qui lui parvint l'année de la défaite de l'Invincible Armada ${ }^{38}$.

Si, par les ambitions de Hideyoshi, la décennie 1580 a constitué un tournant majeur du point de vue de l'évolution politique du Japon et de son positionnement dans le monde, elle a également marqué une rupture dans l'histoire de sa première chrétienté.

En effet, c'est à partir de ces années-là que les jésuites procédèrent à une grande réforme de leur mission japonaise. L'ordonnateur en fut Alexandre Valignano, visiteur des Indes orientales ; c'est à ce titre qu'il séjourna pour une première fois au Japon entre 1579 et 1582. Au programme de l'Italien : la nécessité de s'adapter aux mœurs locales, et notamment de s'initier à la langue japonaise ; la création de séminaires, de collèges et de noviciats, mais aussi de monts-de-piété et d'hôpitaux ; enfin, le projet de développer un clergé indigène. Les ambitions étaient en fait à la hauteur de la menace que faisait encourir à la Société de Jésus l'intérêt grandissant pour l'archipel des autres ordres missionnaires (1582: arrivée informelle au Japon des deux premiers Franciscains ; 1593 : entrée officielle de leurs coreligionnaires qui ouvrent leur propre mission). Valignano prit d'ailleurs la peine de justifier par écrit les « raisons de s'opposer à la venue au Japon d'autres congrégations religieuses » (Sumario, $1583)^{39}$.

Le renforcement de la mission jésuite passa également par la création et l'appropriation du port de Nagasaki, qui devint un comptoir portugais en 1580. Avec l'accord du daimyô Ômura Sumitada, les Jésuites y jouirent pendant sept ans de l'usufruit de la terre, de la gestion administrative de la cité ainsi que des droits d'ancrage des navires portugais (mais Ômura se réserva les taxes sur le commerce international, dix fois plus lucratives); la ville était défendue par une forteresse ; et de très nombreux habitants - pour la plupart japonais, mais on comptait aussi beaucoup de Chinois, de Coréens et d'Européens - étaient chrétiens ${ }^{40}$. Nagasaki devint alors le principal port de commerce international du Japon. C'est là qu'arrivaient les navires lusitaniens chargés de la soie grège de Chine (Macao). Il n'est donc guère étonnant que le général Toyotomi Hideyoshi se soit lui aussi intéressé à ce centre économique. On peut même supposer que l'édit d'expulsion qu'il promulgua à l'encontre des missionnaires en $1587^{41}$, au lendemain de sa victoire définitive sur le Kyûshû, a largement été

\footnotetext{
${ }^{36}$ ASAO Naohiro, «Jûroku seiki kôhan no Nihon. Tôgô sareta shakai he » [Le Japon dans la seconde moitié du XVI ${ }^{\mathrm{e}}$ siècle. Vers une société unifiée], dans Nihon Tsûshi 11-Kinsei 1, Tôkyô, Iwanami shoten, 1993, p. 13.

${ }^{37}$ Ibid., p. 10.

${ }^{38}$ Sur ce projet, voir Charles R. BOXER, op. cit., p. 257-259.

${ }^{39}$ Alexandre VAlignano, Les jésuites au Japon. Relation missionnaire (1583). Traduction, présentation et notes de J. Bésineau, s.j., Paris, Desclée de Brouwer Bellarmin, 1990, p. 127-133.

${ }^{40}$ Voir SENO S. et al., op. cit., p. 125-191.

${ }^{41}$ Ce texte est daté du $19^{\mathrm{e}}$ jour du $6^{\mathrm{e}}$ mois de la $15^{\mathrm{e}}$ année de l'ère Tenshô (24 juillet 1587$)$. Il comporte cinq clauses; la troisième est la plus célèbre : «Jusqu'à présent Sa Seigneurie [c'est-à-dire Hideyoshi] était convaincue du fait que les missionnaires chrétiens gagnaient le cœur des fidèles au moyen de leur science, mais
} 
motivé par le désir de s'emparer de la cité chrétienne. C'est en tout cas ce qu'il fit dès l'année suivante, en reprenant aux Ômura la ville qu'il leur avait d'abord rendue.

On considère d'ordinaire que l'édit de 1587 marque le début de la répression antichrétienne au Japon. Il y aurait un avant et un après 1587. Pour souligner l'importance de la rupture, de nombreux historiens présentent comme un événement considérable la mise à mort publique dix ans plus tard, à Nagasaki, au nom de cet édit, de vingt-six chrétiens européens et japonais (1597). Il est vrai que le geste était sans précédent dans un pays où l'Etat, ayant toujours opté pour le pluralisme religieux, n'avait pour ainsi dire jamais réprimé d'institutions ou d'hommes de religion. Il est aussi exact que ce coup de tonnerre résonna d'autant plus fortement dans le ciel de la chrétientét ${ }^{42}$ que le Japon faisait figure de Terre promise depuis l'époque de Xavier. Cela dit, on peut donner mille exemples de ce que, pendant les trois décennies qui ont suivi leur promulgation, les prétendues mesures «antichrétiennes» de Hideyoshi n'ont quasiment pas été appliquées. Par exemple, bien qu'étant officiellement désigné comme une religion hétérodoxe dans «le pays des kami et des bouddhas ", le christianisme n'a pas été déclaré illicite (jusqu'en 1612); les missionnaires n'ont pas quitté le territoire nippon, mais au contraire ont continué d'y affluer, qu'ils soient jésuites ou, désormais, franciscains, dominicains, augustins; et pendant tout ce temps, Toyotomi Hideyoshi et son successeur Tokugawa Ieyasu n'ont montré aucune gêne pour continuer à accueillir à bras ouverts dans leurs palais les pères étrangers. Autrement dit, pour comprendre les raisons de l'exécution de ces vingt-six malheureuses personnes, il faut minimiser les motivations idéologiques de Hideyoshi. Le fait que la plupart des victimes étaient rattachées à l'ordre pro-espagnol des franciscains suggère plutôt que l'exécution s'inscrivit dans les relations diplomatiques tendues que ce général avait engagées depuis plusieurs années avec Manille, qui en 1597 encore s'obstinait à refuser de lui payer tribut alors qu'il venait de décider de lancer une deuxième campagne contre la Corée (1597-1598) et qu'il avait besoin de fonds importants. L'ambassadeur de Manille et franciscain Blazquez Pedro Bautista faisait partie des vingt-six « martyrs ».

Le décret de 1587 ne fut jamais abrogé. C'est qu'en maintenant une pression constante sur les étrangers on s'assurait à peu de frais de leur soumission, tout en continuant à bénéficier de leurs services : on pouvait compter sur eux pour les transactions commerciales internationales (que Hideyoshi et Ieyasu ont essayé, chacun à leur manière, de contrôler), mais aussi pour la création éventuelle de nouvelles routes de commerce en direction de la Nouvelle-Espagne et l'apport de connaissances techniques (mines, construction navale) ${ }^{43}$.

Mais le plus intéressant n'est-il pas que, dans ce climat très tendu, la communauté chrétienne du Japon connut un véritable d'âge d'or ? Les décennies 1580-1610 représentent en effet les Trente Glorieuses de l'histoire chrétienne du Japon, au cours desquelles le nombre de conversions augmenta de manière significative ${ }^{44}$, non seulement dans les centres traditionnels du prosélytisme catholique, mais aussi dans des régions jusqu'alors peu touchées par les missionnaires, soit les lointaines provinces de l'Est et du Nord. Ces années furent aussi

en réalité, ils détruisent la Loi bouddhique du Japon en utilisant les moyens que l'on vient de voir, ce qui est intolérable. Par conséquent, les missionnaires chrétiens étrangers ne doivent plus résider sur le sol japonais et doivent se préparer au départ et rentrer dans leur pays dans un délai de vingt jours à compter d'aujourd'hui. D'ici là, on ne doit pas tolérer que quelqu'un s'en prenne à eux. »

${ }^{42}$ Par exemple, d'après le Journal du chroniqueur indien Domingo Chimalpahin, la nouvelle de l'exécution fut rapportée dans l'année à Mexico ; la ville accueillit fin 1598 une partie des dépouilles des suppliciés, cf. Serge GRUZINSKI, Les quatre parties du monde. Histoire d'une mondialisation, Paris, Editions de La Martinière, 2004, p. 25.

${ }^{43}$ Cf. AsAo Naohiro, «Sakoku sei no seiritsu » [La mise en place du système de la fermeture du pays], dans Asao Naohiro chosakushû, 3. Shôgun kenryoku no sôshutsu, Tôkyô, Iwanami shoten, 2004, p. 296-300.

${ }^{44} \mathrm{D}$ 'après les sources jésuites de l'époque, il y aurait eu 150000 chrétiens japonais au début des années 1580 , et 300000 dans les années 1610. 
celles où s'épanouirent une littérature chrétienne ou à usage apostolique (une centaine de titres publiés par les presses jésuites : manuels de langue, livres de doctrine...) ainsi qu'un art nippo-européen (tel qu'il se manifesta par exemple dans une nouvelle peinture de style occidental et les pittoresques paravents des barbares du sud $)^{45}$. Ce fut, encore, le temps du plein épanouissement de Nagasaki, dont la population croissante était pour une bonne part de confession chrétienne (y compris les cadres municipaux) et animaient les diverses églises catholiques de la ville. En somme, c'est sur un mode tout à fait original qu'au Japon, au cours de la première mondialisation, évangélisation (européenne) rima avec colonisation (japonaise).

\section{Troisième époque : l'impossible évangélisation (à partir de 1614) - conclusion}

En promulguant l'édit de 1587, le général Toyotomi Hideyoshi avait voulu rappeler aux missionnaires qu'elle était la place à laquelle ils pouvaient prétendre au sein de son pays celle de partenaires dociles. En rédigeant de nouveaux textes de même teneur (lettre au gouverneur des Philippines de 1605, puis édits de 1612 et 1614), son successeur Tokugawa Ieyasu adopta la même attitude, mais dans un contexte historique nouveau, celui de l'érosion de l'image de marque de missionnaires que l'on avait jusqu'alors assez bien considérés. De fait, tout concourait à flétrir le prestige des clercs étrangers, ou du moins à affaiblir leur position. Ainsi, d'un point de vue économique, il devenait chaque jour plus évident que le rôle qu'ils jouaient était moins indispensable qu'autrefois : d'une part, parce que l'entrée en scène des marchands hollandais et britanniques (qui, eux, ne se souciaient pas de prosélytisme) ${ }^{46}$ sonnait de facto le glas du monopole commercial des Portugais et des jésuites ; d'autre part, parce qu'ayant appris à parler la langue de l'autre un certain nombre d'étrangers et de Japonais pouvaient désormais se dispenser des services d'interprétariat des missionnaires ; enfin, parce qu'à partir de 1601 le nouveau système de licences internationales mis en place par les Tokugawa donna aux Japonais le moyen de prendre eux-mêmes en main les transactions commerciales entre le Japon et l'Asie orientale (hors Chine). Ajoutons à cela le fait que dans les années 1610 plusieurs affaires concernant des chrétiens firent scandale ${ }^{47}$, qui alimentèrent fortement les campagnes de calomnie anti-ibéro-catho-jésuites des Européens du Nord-Ouest qui fréquentaient eux aussi les élites japonaises - à l'époque Tokugawa Ieyasu écoutait très attentivement ce que lui disait son conseiller britannique William Adams (1564$1620)^{48}$. Enfin, le développement d'une pensée japonaise plus sûre d'elle-même fit beaucoup

\footnotetext{
45 Sur les aspects culturels des premières relations nippo-européennes, voir par exemple le catalogue d'exposition intitulé Art Namban. Les Portugais au Japon / Nambankunst. Portugezen in Japan, Bruxelles, Musées royaux d'Art et d'Histoire-Europalia 89, 1989, et Jacques Proust, L'Europe au prisme du Japon, XVI ${ }^{-}$ XVIII ${ }^{e}$ siècle. Entre humanisme, Contre-Réforme et Lumières, Paris, Albin Michel, 1997. La bibliographie japonaise sur la question est très importante, cf. N. KOUAME, «Japon : le "siècle chrétien", son historiographie et ses lieux de mémoire », Histoire \& Missions chrétiennes, n 4 , 2007, p. 171-182.

46 1609 : la Compagnie hollandaise des Indes orientales ouvre un comptoir à Hirado (Kyûshû) ; 1613 : la Compagnie anglaise des Indes orientales y installe elle aussi un comptoir.

${ }^{47} 1610$ : Incident du navire Madre de Deus ; 1612 : Incident Okamoto Daihachi ; 1613 : exécution publique à Kyôto d'un fraudeur chrétien.

${ }^{48}$ Envoyé avec quatre autres navires en Extrême-Orient par la nouvelle république des Provinces-Unies, le Liefde s'échoua dans la province de Bungo, à Kyûshû, en l'an 1600. Williams Adams, de nationalité britannique, faisait partie des quelques rescapés du naufrage. Cétait le premier Anglais à fouler le sol du Japon. Conseiller personnel de Tokugawa Ieyasu qu'il rencontra sitôt arrivé au Japon, il diffusa des connaissances européennes auprès de grands commis du régime (géographie mathématiques, science de la navigation...). Adams avait un intérêt personnel à dénigrer les Portugais et leurs comparses missionnaires : dès 1613 il avait obtenu un contrat de la Compagnie anglaise des Indes orientales. Ayant aussi bénéficié de licences commerciales shôgunales, il
} 
pour dévaloriser le message et la vocation évangéliques des pères. En effet, au tournant des $\mathrm{XVI}^{\mathrm{e}}$ et $\mathrm{XVII}^{\mathrm{e}}$ siècles, les Japonais approfondirent leur réflexion sur leur identité nationale en mobilisant toutes les religions traditionnelles : shintô, bouddhisme, voie du Yin et du Yang, confucianisme. Cette méditation qui fut notamment menée par l'école officielle du «shintô des Yoshida » (jap. Yoshida shintô) se répandit sous la forme d'une vulgate selon laquelle le Japon était, et devait rester, un pays protégé par les dieux du shintô et par les bouddhas. Cette idée se retrouve très explicitement formulée dans les deux grands textes « antichrétiens » de Toyotomi Hideyoshi (1587) et de Tokugawa Ieyasu $(1614)^{49}$.

Il reste que la promulgation de ces textes obéit alors sans doute davantage à un besoin politique immédiat qu'à une réelle volonté d'éradiquer le christianisme. Nous avons déjà dit ce que l'on pouvait penser de l'édit de Hideyoshi ; en ce qui concerne ceux de $1614^{50}$, qui furent suivis à la lettre puisque les missionnaires et certaines de leurs ouailles japonaises de haut rang se résignèrent cette fois-ci à l'exil (le départ de Takayama Ukon est resté célèbre), Ieyasu semble surtout avoir cherché à se rapprocher des ennemis des «papistes », soit les Néerlandais et les Britanniques qui étaient à même de lui fournir le matériel de guerre indispensable à la survie de son nouveau régime : essentiellement canons, mousquets et plomb. C'est qu'au début des années 1610, le fils de Toyotomi Hideyoshi, Hideyori (15931615), se dressait encore comme le successeur légitime de son père disparu en 1598. Grâce au recours des mousquets et d'une artillerie de fabrication étrangère pendant la grande campagne militaire qu'il mena en 1614-1615 contre la forteresse d'Ôsaka où s'était réfugiée l'armée des nombreux partisans de Hideyori ${ }^{51}$, Ieyasu fut définitivement débarrassé de cette menace politique. On n'exagérera donc pas l'importance historique des annonces faites en 1614 à l'égard des chrétiens du Japon.

Mais si l'on admet que la proscription fut alors plus tactique qu'idéologique, et qu'elle n'était pas nécessairement conçue comme irrévocable, on peut aussi penser que les mesures répressives qui s'égrainèrent par la suite furent moins la confirmation d'une position résolument antichrétienne de la part des shôgun Tokugawa, que le résultat de leur constatation que, finalement, la stratégie de la proscription était plus avantageuse que celle de la tolérance.

entreprit des voyages d'affaires à Okinawa, au Siam, au Annam et au Tonkin. Enfin, il contribua également à l'ouverture des relations commerciales entre la Hollande et le Japon.

49 En ce qui concerne le contenu proprement théologique du texte rédigé en 1614 par Sûden sur ordre d'Ieyasu (voir note suivante), l'historien Takagi Shôsaku a montré que le moine zen avait beaucoup emprunté à la Précieuse chronique des bonnes relations entretenues avec nos voisins (1466) de son coreligionnaire Zuikei Shûhô (1391-1473) et aux idées exprimées dans l'Anthologie des doctrines du shintô unique (seconde moitié du $\mathrm{XV}^{\mathrm{e}}$ s.) du lettré Yoshida Kanetomo (1435-1511). Cf. TAKAGI Shôsaku, Shôgun kenryoku to tennô. Hideyoshi Ieyasu no shinkoku kan [Le pouvoir shôgunal et les empereurs. Le pays des dieux selon Hideyoshi et Ieyasu], Tôkyô, Aogi shoten, 2003, p. 30-39. Il n’y eut donc pas de véritable innovation dans le discours théorique des Tokugawa, si ce n'est le fait - très important - que ce discours fut au service d'une toute nouvelle orthodoxie antichrétienne.

${ }^{50}$ Voir note précédente. En réalité, les Tokugawa avaient fait rédiger deux textes de nature bien distincte en cette fin de $12^{\mathrm{e}}$ mois de la $18^{\mathrm{e}}$ année de l'ère Keichô : le premier, rédigé le $19^{\mathrm{e}}$ jour (soit le 28 janvier 1614), prenait des dispositions très concrètes à l'égard des chrétiens : rassembler à Nagasaki tous les missionnaires dispersés dans l'archipel, détruire les églises, faire apostasier les fidèles ; le second, rédigé par le moine Ishin Sûden dans la nuit du 22 au $23^{\mathrm{e}}$ jour, développait un discours politique et idéologique sur le thème de l'identité japonaise : qu'est-ce qui caractérise le Japon, en quoi ce pays est-il différent des autres, que faire pour préserver son intégrité face aux menaces que font peser sur lui d'autres nations?

${ }^{51} \mathrm{Au}$ début d'une première campagne dite d'hiver, le principal stratège des Toyotomi présent dans le château d'Ôsaka, Sanada Yukimura (1567-1615), remporta les premiers combats parce qu'il sut utiliser massivement la puissance de feu des mousquets. Mais dans un second temps, Ieyasu utilisa contre la forteresse des canons qui étaient de fabrication japonaise, anglaise et hollandaise; il eut aussi recours aux services de cannoniers de nationalité hollandaise. Le déploiement de cette artillerie convainquit les Hideyoshi qu'il leur fallait s'engager dans des négociations; la trève permit à Ieyasu de faire combler les fossés du château au grand dam de ses ennemis. Cf. KIKUCHI Michito, «Ôsaka fuyu natsu no jin shiwa » [A propos des deux sièges d'hiver et d'été d'Ôsaka], dans OzAKI Hokki (dir.), Tokugawa sandai naruhodo jiten, Tôkyô, Tôkyôdô shuppan, 1999, p. 72-76. 
Une telle hypothèse pourrait expliquer que pendant longtemps les seigneurs régionaux du Japon appliquèrent les mesures promulguées par la capitale militaire d'Edo avec un zèle très inégal : on réprima là où l'on avait intérêt à le faire : plusieurs daimyô de Kyûshû se montrèrent particulièrement sévères, car ce fut pour eux une manière d'exprimer leur soumission nouvelle aux Tokugawa ${ }^{52}$; les seigneurs apanagistes d'Owari (dans le centre du pays) attendirent pour leur part la décennie 1660 pour se lancer dans une rigoureuse campagne antichrétienne; et dans la partie d'Ezo contrôlée par les Matsumae les grandes années de prosélytisme furent justement la période $1610-1630^{53}$ !

En outre, chacun sait que les dispositions antichrétiennes s'accompagnèrent de mesures plus strictement politico-économiques, celles-là même qui finirent par assurer aux Tokugawa le monopole des relations extérieures, qu'il s'agisse de commerce ou de diplomatie. L'édit de 1616 fut le premier d'une longue série : de manière à contrôler les arrivées des étrangers, et notamment des missionnaires interdits de séjour depuis 1614, on restreignit cette année-là les ports d'accès au Japon à Nagasaki et Hirado ; l'idée était probablement d'inspiration chinoise, puisque autrefois l'Empire du Milieu avait imposé aux Japonais le port de Ningbo comme unique porte d'entrée à son territoire. Bien d'autres mesures suivirent, selon cette double logique religieuse et politico-économique, qui finalement aboutirent à la «fermeture du pays » vers $1640^{54}$.

Pour notre propos, il est très important de souligner que ce processus de repli fut rendu possible par le fait qu'il ne se trouva personne outre-mer pour s'y opposer réellement : les quelques délégations envoyées par le Vieux Monde pour demander la réouverture du Japon furent fermement éconduites; les Néerlandais acceptèrent d'être confinés à Dejima ; et la poignée de catholiques désireux de poursuivre le travail évangélique, ou d'être " martyrisés », agit en ordre dispersé. Ici, au moment où les puissances étrangères durent faire le choix d'accepter ou de refuser les conditions imposées, il est fort probable que la réputation acquise par les Japonais au cours du $\mathrm{XVI}^{\mathrm{e}}$ siècle joua en leur faveur. En tout cas, dans la seconde moitié du $\mathrm{XVII}^{\mathrm{e}}$ siècle, l'archipel retrouva l'isolement (relatif) dans lequel il avait vécu pendant un millénaire, même s'il était désormais condamné à rester constamment sur ses gardes.

\footnotetext{
${ }^{52}$ Asao Naohiro évoque la «fonction de fumie » que remplit la répression antichrétienne lorsqu'elle fut le fait des seigneurs du Sud-Ouest, faisant là référence aux fameuses cérémonies par lesquelles, à partir de la fin des années 1620, les Japonais suspectés d'hérésie d'abord, puis l'ensemble de la population du pays, furent amenés à piétiner des «images à fouler » (fumie, représentant la Vierge, le Christ...) pour démontrer qu'ils n'étaient pas chrétiens et prouver leur loyauté à l'égard du régime. Cf. ASAO Naohiro, «Sakoku sei no seiritsu », op. cit., p. 305.

${ }^{53}$ Sur la chronologie de la répression antichrétienne des Tokugawa, cf. MURAI Sanae, Kirishitan kinsei to minsh $\hat{u}$ no shûkyô [L'interdiction du premier christianisme et les religions populaires], Tôkyô, Yamakawa shuppansha, 2002, p. 6-28. Sur la répression du christianisme et le problème de l'attitude fondamentale des Tokugawa à l'égard $\mathrm{du}$ fait religieux, voir notre article, N. KouAME, «L'État des Tokugawa et la religion: entre intransigeance et tolérance (XVII ${ }^{\mathrm{e}}$-XIX ${ }^{\mathrm{e}}$ siècles) », Archives de Sciences Sociales des Religions, $\mathrm{n}^{\circ} 137,2007, \mathrm{p}$. 107-123.

${ }^{54} 1624$ : expulsion des Espagnols ; 1635 : interdiction faite aux Japonais de rentrer au Japon ou de sortir du territoire ; 1639 : expulsion des Portugais ; 1641 : les Néerlandais installés à Hirado sont transférés à Dejima, un îlot construit au large de la ville de Nagasaki. Les Britanniques avaient fermé leur comptoir et quitté volontairement le Japon en 1623 ; les Chinois étaient installés dans un quartier spécial de Nagasaki ; les Coréens gardaient contact avec le Japon via l'archipel de Tsushima.
} 